\title{
A note on Global Gauge Anomalies
}

\author{
Roberto Catenacci \\ Dipartimento di Scienze e Tecnologie Avanzate \\ Università del Piemonte Orientale "A.Avogadro" - Alessandria - Italy \\ Sezione I.N.F.N. di Pavia - Pavia - Italy \\ Augusto Lena \\ via A.Lombardi 8 - Bologna - Italy
}

abstract - Using methods of group cohomology the definitions of global and local anomalies are revisited taking into account the fact that the action of the gauge group on the space of connections is not free. The global $S U(2)$ anomaly appears as an abelian one.

\section{1 - Introduction}

In his '82 paper [1] Witten clarified the problems arising in quantizing a $S U(2)$ gauge theory with an odd number of chiral fermion doublets by showing the mathematical inconsistency of the theory (in an odd topological sector). This was the starting point of global anomalies, a well known, completely understood subject.

Nevertheless some minor questions remain, and in this note we revisit one of them; in particular we observe that the interpretation of the $S U(2)$ anomaly depends on the "gauge group" that one takes in order to have a free action on the space of connections.

When one takes into account the action of the center the global gauge anomaly appears to be an abelian one.

The usual picture is that the origin of the anomalies problem is the lacking of gauge invariance of the effective action; local anomalies arise when considering "infinitesimal" gauge transformation, while global anomalies are connected with "large" transformation (i.e. not in the connected component of the identity).

The general framework for testing the possible occurrence of anomalies in field theories can be constructed in terms of the theory of group actions on line bundles (see e.g.[2] and its references).

In this note we briefly review this topological construction through the application of methods of group cohomology.

The starting point is the concept of $\mathcal{G}$-line bundle over a principal $\mathcal{G}$-bundle $P \stackrel{\pi}{\rightarrow} M$. In physical applications $P$ is the configuration space, while $\mathcal{G}$ is the invariance group of the theory and the effective action $\mathcal{Z}(p)$ is a section of this $\mathcal{G}$-line bundle.

The relevant cohomology group in which the anomalies live is interpreted as the kernel of the map induced in (integer) cohomology by the projection map of the principal fibration $P$. Local and global anomalies are split via the quotient fibration $P / \mathcal{G}_{0}$ (where $\mathcal{G}_{0}$ is the identity connected component of $\mathcal{G}$ ) : $P \stackrel{l}{\rightarrow} P^{\prime}=P / \mathcal{G}_{0} \stackrel{g}{\rightarrow} M=P^{\prime} / \pi_{0}(\mathcal{G})$. Then some spectral sequences analysis applies to describe the "anomalies groups" and the "anomalies sequence".

In gauge theories $P$ should be the space of connections, $\mathcal{G}$ the gauge group and the $\mathcal{G}$-line bundle the determinant index bundle of the Dirac operator actings on fermions (see also [3]). 
However the action of $\mathcal{G}$ on $P$ is not free and in order to have a principal bundle two different construction can be introduced (see e.g.[4]).

One can either reduce the space of all connections to the space of irreducible connections and the gauge group to its quotient by the center of $S U(2)\left(\mathbf{Z}_{2}\right)$, or simply reduce the gauge group to the group of pointed gauge transformation.

Note that in our case of a $S U(2)$ theory over $S^{4}$ with the gauge fields in a non trivial topological sector, all connections are irreducible (see [5]).

Witten's point of wiew was to use the pointed gauge transformation, but the other one is perfectly equivalent if the action of the center on the determinant bundle is taken into account (note that the center acts trivially on the connections).

\section{2 - Group Cohomology}

If $\mathcal{G}$ is a group and $\mathcal{M}$ a right $\mathcal{G}$-module, the group cohomology with coefficients in $\mathcal{M}, H^{*}(\mathcal{G}, \mathcal{M})$, is the cohomology of the complex $C^{*}(\mathcal{G}, \mathcal{M})$, where $C^{n}(\mathcal{G}, \mathcal{M})$, the module of n-cochains, is the abelian group of maps from $\mathcal{G} \times \mathcal{G} \times \ldots \times \mathcal{G}$ to $\mathcal{M}$. The coboundary operator, $d^{n}: C^{n}(\mathcal{G}, \mathcal{M}) \rightarrow C^{n+1}(\mathcal{G}, \mathcal{M})$, is:

$$
d^{n} F\left(g_{1} \ldots g_{n+1}\right)=F\left(g_{2} \ldots g_{n+1}\right) g_{1}+\sum_{1}^{n}(-1)^{i} F\left(g_{1} \ldots g_{i} g_{i+1} \ldots g_{n+1}\right)+(-1)^{n+1} F\left(g_{1} \ldots g_{n}\right)
$$

The module of n-cochains $C^{n}(\mathcal{G}, \mathcal{M})$ is a $\mathcal{G}$-module with a $\mathcal{G}$-action given by:

$$
(F g)\left(g_{1} \ldots g_{n}\right)=F\left(g^{-1} g_{1} g \ldots g^{-1} g_{n} g\right) g
$$

This action is trivial on $H^{*}(\mathcal{G}, \mathcal{M})$.

When $\mathcal{H}$ is a normal subgroup of $\mathcal{G}$, one has an exact sequence:

$$
0 \rightarrow H^{1}\left(\mathcal{G} / \mathcal{H}, \mathcal{M}^{\mathcal{H}}\right) \stackrel{\text { inf }}{\rightarrow} H^{1}(\mathcal{G}, \mathcal{M}) \stackrel{\text { res }}{\rightarrow} H^{1}(\mathcal{H}, \mathcal{M})^{\mathcal{G}} \stackrel{T}{\rightarrow} H^{2}\left(\mathcal{G} / \mathcal{H}, \mathcal{M}^{\mathcal{H}}\right) \stackrel{\text { inf }}{\rightarrow} H^{2}(\mathcal{G}, \mathcal{M})
$$

where, for a $\mathcal{G}$-module $\mathcal{N}$, we have denoted by $\mathcal{N}^{\mathcal{G}}$ the $\mathcal{G}$-invariant elements. The homomorphisms res and inf are, respectively, the restriction to $\mathcal{H}$ of the cocycles of $\mathcal{G}$, and the inflation, i.e. the composition of the cocycles of $\mathcal{G} / \mathcal{H}$ with the projection $p: \mathcal{G} \rightarrow \mathcal{G} / \mathcal{H}$. The homomorphism $T$ is the transgression.

When $\mathcal{H}$ is a finite index normal subgroup of $\mathcal{G}$, there exists a homomorphism, called corestriction, going in the opposite direction: cor $: H^{*}(\mathcal{H}, \mathcal{M}) \rightarrow H^{*}(\mathcal{G}, \mathcal{M})$. It is the homomorphism defined, in dimension zero (where $\operatorname{cor}: \mathcal{M}^{\mathcal{H}} \rightarrow \mathcal{M}^{\mathcal{G}}$ ), by:

$$
\operatorname{cor}(m)=\sum_{c \in \mathcal{G} / \mathcal{H}} m \bar{c}
$$

where, for each coset $c \in \mathcal{G} / \mathcal{H}$ choose, once and for all, a representative $\bar{c}$ requiring that for $c=\mathcal{H}, \bar{c}=1$. Note that, for $g \in \mathcal{G}, \bar{c} g$ and $\overline{c g}$ are such that $\bar{c} g \overline{c g}^{-1} \in \mathcal{H}$. The definition of cor in dimension one is:

$$
(\text { coru })(g)=\sum_{c \in \mathcal{G} / \mathcal{H}} u\left(\bar{c} g \overline{c g}^{-1}\right) \bar{c}
$$

An important property of cor is that the two compositions cor.res and res.cor are both the multiplication by $n=(\mathcal{G}: \mathcal{H})$. It follows that, for any $m$ different from zero, $n a=0$ for any $a \in H^{m}\left(\mathcal{G} / \mathcal{H}, \mathcal{M}^{\mathcal{H}}\right)$. 


\section{3 - The Anomalies Sequence}

The elementary properties of group cohomology briefly recalled in the previous section turn out to be well suited for a description of anomalies. The only point is to find the "relevant" $\mathcal{G}$-module $\mathcal{M}$.

The interpretation of the effective action $\mathcal{Z}$ as a section of a $\mathcal{G}$-line bundle over $P$, gives naturally that $\mathcal{M}=\mathbf{C}^{*}(P)$, the right $\mathcal{G}$-module of the non vanishing functions from $P$ to $\mathbf{C}$, with the natural action $(f g)(p)=f(p g)$. As usual, we switch to a multiplicative notation for $\mathbf{C}^{*}$ and, therefore, for the cohomology.

A one-cochain $F: \mathcal{G} \rightarrow \mathbf{C}^{*}(P)$, putting $f(p, g)=F(g)(p)$, gives a map $f: P \times \mathcal{G} \rightarrow \mathbf{C}^{*}$, and the cocycle condition become $f\left(p, g_{1} g_{2}\right)=f\left(p g_{1}, g_{2}\right) f\left(p, g_{1}\right)$. The cohomology group $H^{1}\left(\mathcal{G}, \mathbf{C}^{*}(P)\right)$ represents, geometrically, the group of $\mathcal{G}$-isomorphism classes of topologically trivial $\mathcal{G}$-lines bundles over $P$, that is the anomalies. This can be seen from the exact sequence:

$$
1 \rightarrow H^{1}\left(\mathcal{G}, \mathbf{C}^{*}(P)\right) \rightarrow H^{2}(M, \mathbf{Z}) \stackrel{\pi^{*}}{\rightarrow} H^{2}(P, \mathbf{Z})
$$

where the first (injective) arrow is given by $f \rightarrow P \times{ }_{f} \mathbf{C}$ (i.e. we identify $(p, c)$ and $(p g, f(p, g) c)$ ).

These $\mathcal{G}$-isomorphism classes represent the anomalies in the sense that in perturbative field theory one first defines the effective action $\mathcal{Z}(p)$; the obstruction to extending this functional to the whole $\mathcal{G}$-orbit is given by the non triviality of $f$. In fact the action of $\mathcal{G}$ on $\mathcal{Z}$ is represented by $\mathcal{Z}(p g)=f(p, g) \mathcal{Z}(p)$.

When the group $\mathcal{G}$ is not connected, one could find "global anomalies", that is trivial $\mathcal{G}_{0}$-cocycles that extends non trivially to $\mathcal{G}$. The non trivial (and $\mathcal{G}$-invariant) $\mathcal{G}_{0}$-cocycles are called "local anomalies".

Putting $\mathcal{H}=\mathcal{G}_{0}$ and observing that $\mathbf{C}^{*}(P)^{\mathcal{G}_{0}}=\mathbf{C}^{*}\left(P^{\prime}\right)$, the exact sequence of the previous section gives the "anomalies sequence":

$$
1 \rightarrow H^{1}\left(\pi_{0}(\mathcal{G}), \mathbf{C}^{*}\left(P^{\prime}\right)\right) \stackrel{\text { inf }}{\rightarrow} H^{1}\left(\mathcal{G}, \mathbf{C}^{*}(P)\right) \stackrel{\text { res }}{\rightarrow} H^{1}\left(\mathcal{G}_{0}, \mathbf{C}^{*}(P)\right)^{\mathcal{G}} \stackrel{T}{\rightarrow} H^{2}\left(\pi_{0}(\mathcal{G}), \mathbf{C}^{*}\left(P^{\prime}\right)\right)
$$

The geometrical interpretation in terms of line bundles over $P$, applied to the factorisation $P \stackrel{l}{\rightarrow} P^{\prime}=$ $P / \mathcal{G}_{0} \stackrel{g}{\rightarrow} M=P^{\prime} / \pi_{0}(\mathcal{G})$, gives:

$$
1 \rightarrow H^{1}\left(\mathcal{G}_{0}, \mathbf{C}^{*}(P)\right) \rightarrow H^{2}\left(P^{\prime}, \mathbf{Z}\right) \stackrel{l^{*}}{\rightarrow} H^{2}(P, \mathbf{Z})
$$

and

$$
1 \rightarrow H^{1}\left(\pi_{0}(\mathcal{G}), \mathbf{C}^{*}\left(P^{\prime}\right)\right) \rightarrow H^{2}(M, \mathbf{Z}) \stackrel{g^{*}}{\rightarrow} H^{2}\left(P^{\prime}, \mathbf{Z}\right)
$$

These sequences identify $H^{1}\left(\pi_{0}(\mathcal{G}), \mathbf{C}^{*}\left(P^{\prime}\right)\right)$ with the global anomalies and the $\mathcal{G}$-invariant elements of $H^{1}\left(\mathcal{G}_{0}, \mathbf{C}^{*}(P)\right)$ with the local anomalies.

Note that, if $\pi_{0}(\mathcal{G})$ is finite, the anomalies sequence implies that, if $H^{2}\left(\pi_{0}(\mathcal{G}), \mathbf{C}^{*}\left(P^{\prime}\right)\right)$ is trivial, the map res is surjective. Moreover cor is injective. This means that the only torsion elements in the anomaly group are the global anomalies. All local anomalies, in this case, can be detected via the family index theorem and represented by functionals on the space $P$.

The topological interpretation of the first cohomology group of $\mathcal{G}$ with values in the $\mathcal{G}$-module $\mathbf{C}^{*}(P)$ gives a more explicit description of anomalies in terms of the topology of $\mathcal{G}$ and $P$.

For the local anomalies, $H^{1}\left(\mathcal{G}_{0}, \mathbf{C}^{*}(P)\right)$, one can apply the low dimensional exact cohomology sequence of the bundle $P \rightarrow P^{\prime}$. In this case (recall that $\mathcal{G}_{0}$ is, by definition, a connected group) the Leray spectral sequence gives, in absence of monodromy, an exact sequence. The result is:

$$
0 \rightarrow H^{1}\left(P^{\prime}, \mathbf{Z}\right) \rightarrow H^{1}(P, \mathbf{Z}) \rightarrow H^{1}\left(\mathcal{G}_{0}, \mathbf{Z}\right) \rightarrow H^{2}\left(P^{\prime}, \mathbf{Z}\right) \stackrel{l^{*}}{\rightarrow} H^{2}(P, \mathbf{Z})
$$


From this,

$$
H^{1}\left(\mathcal{G}_{0}, \mathbf{C}^{*}(P)\right)=\operatorname{kerl}^{*}=H^{1}\left(\mathcal{G}_{0}, \mathbf{Z}\right) / H^{1}(P, \mathbf{Z})
$$

In the case of gauge theories, where $P$ is the space of connections, all the cohomology of $P$ is trivial and we find:

$$
H^{1}\left(\mathcal{G}_{0}, \mathbf{C}^{*}(P)\right)=H^{1}\left(\mathcal{G}_{0}, \mathbf{Z}\right)=H^{2}\left(P^{\prime}, \mathbf{Z}\right)
$$

The last group is the group of lines bundles over $P^{\prime}$; the effective action is a section of the determinant line bundle of the Dirac chiral operator over $P$. This line bundle is $\mathcal{G}_{0}$ trivial if the corresponding bundle over $P^{\prime}$ has vanishing Chern class. The local anomaly cancellation is controlled by the family index theorem that computes precisely this class.

\section{4 - The $S U(2)$ case.}

In this section we study the case of $S U(2)$ from two point of view.

In the "usual one", we denote $\mathcal{G}^{*}$ the pointed gauge group and $\mathcal{G}_{0}^{*}$ its identity connected component. The relevant fibration is $P \rightarrow P^{\prime}=P / \mathcal{G}_{0}^{*} \rightarrow M=P^{\prime} / \pi_{0}\left(\mathcal{G}^{*}\right)$ where $P$ is the space of connections and $\pi_{0}\left(\mathcal{G}^{*}\right)=\mathbf{Z}_{2}$.

Since $P$ is contractible, $\pi_{2}\left(P^{\prime}\right)=\pi_{1}\left(\mathcal{G}_{0}^{*}\right), \pi_{1}\left(P^{\prime}\right)=\pi_{0}\left(\mathcal{G}_{0}^{*}\right)=0$ and $\pi_{0}\left(P^{\prime}\right)=0$.

This means (by Hurewicz) that $H_{2}\left(P^{\prime}, \mathbf{Z}\right)=\pi_{1}\left(\mathcal{G}_{0}^{*}\right)=\mathbf{Z}_{2}$.

From universal coefficients theorem we find $H^{2}\left(P^{\prime}, \mathbf{Z}\right)=0$ and $H^{2}(M, \mathbf{Z})=\mathbf{Z}_{2}$.

Infact $H^{2}\left(P^{\prime}, \mathbf{Z}\right)=$ Free $_{2}\left(P^{\prime}, \mathbf{Z}\right) \oplus \operatorname{Tor}_{1}\left(P^{\prime}, \mathbf{Z}\right)$ and $H^{2}(M, \mathbf{Z})=\operatorname{FreeH}_{2}(M, \mathbf{Z}) \oplus \operatorname{TorH}_{1}(M, \mathbf{Z})$.

Now recall that $H_{1}(M, \mathbf{Z})=\pi_{0}\left(\mathcal{G}^{*}\right)=\mathbf{Z}_{2}$ and that $H^{2}(M, \mathbf{Z})$ must be a torsion group due to res $\cdot$ cor $=2$.

This gives the "usual" results: local anomalies are absent and there migth be a global anomaly whose square is one. Of course, this "virtual" global anomaly must be computed as Witten did (our discussion can not distinguish between +1 and -1 in $\mathbf{Z}_{2}$ ).

From the other point of view, we consider the group $\overline{\mathcal{G}}=\mathcal{G} / \mathcal{Z}$, where $\mathcal{Z}$ is the center of $G$ (in our case $\mathcal{Z}=\mathbf{Z}_{2}$.

For odd topological charges (see [5]) we have $\pi_{0}(\overline{\mathcal{G}})=0$ : global anomalies are absent.

Also local anomalies are absent: from the homotopy of the fibration $\overline{\mathcal{G}} \rightarrow P \rightarrow M=P / \overline{\mathcal{G}}$ we have $\pi_{1}(M)=0=H_{1}(M, \mathbf{Z})$ and $H_{2}(M, \mathbf{Z})=\pi_{2}(M)=\pi_{1}(\overline{\mathcal{G}})$. Moreover from the fibration $\mathbf{Z}_{2} \rightarrow \mathcal{G} \rightarrow \overline{\mathcal{G}}$ one can conclude that $\pi_{1}(\overline{\mathcal{G}})$ is a torsion group and, finally, $H^{2}(M, \mathbf{Z})=\operatorname{FreeH}_{2}(M, \mathbf{Z}) \oplus \operatorname{TorH}_{1}(M, \mathbf{Z})=0$.

Of course this is not the end of the story; the center $\mathbf{Z}_{2}$ acts on the determinant bundle with a scalar action of weight equal to the ordinary index of the Dirac operator (see see e.g. [4] p.189). This means that if this index is odd, as is in the Witten case of odd topological sector, we have a topologically trivial line bundle with a non-trivial group action, i.e. a anomaly.

This anomaly is not a global anomaly in the usual sense but is more similar to a discrete abelian chiral one.

Note that in this case no use is made of the family index.

\section{Acknowledgements}


The researches reported are supported by MURST $40 \%$ project Metodi geometrici e probabilistici in fisica matematica.

\section{References}

[1] E.Witten, Phys.Lett. 117B, 324-328, 1982

[2] R.Catenacci and G.P.Pirola, Lett. Math. Phys. 19, 45-51, 1990

[3] R.Catenacci, G.P.Pirola, M.Martellini and C.Reina Phys.Lett. 172B, 223-226, 1986

[4] S.K.Donaldson and P.B.Kronheimer, The Geometry of Four-Manifolds, Oxford Mathematical Monographs, Clarendon Press, Oxford 1990

[5] I.M.Singer, Commun.math.phys. 60, 8-13, 1978 